\title{
On the Connection between Flap Side-Edge Noise and Tip Vortex Dynamics
}

\author{
$\begin{array}{lll}\text { D. Casalino* } & \text { A. Hazir } & \text { E. Fares } \\ \ddagger & \text { B. Duda }\end{array}$ \\ Exa GmbH, Curiestrasse 4, Stuttgart, 70563, Germany \\ M. R. Khorrami ${ }^{\S}$ \\ NASA Langley Research Center, Hampton, 23681, Virginia
}

\begin{abstract}
The goal of the present work is to investigate how the dynamics of the vortical flow about the flap side edge of an aircraft determine the acoustic radiation. A validated latticeBoltzmann CFD solution of the unsteady flow about a detailed business jet configuration in approach conditions is used for the present analysis. Evidence of the connection between the noise generated by several segments of the inboard flap tip and the aerodynamic forces acting on the same segments is given, proving that the noise generation mechanism has a spatially coherent and acoustically compact character on the scale of the flap chord, and that the edge-scattering effects are of secondary importance. Subsequently, evidence of the connection between the kinematics of the tip vortex system and the aerodynamic force is provided. The kinematics of the dual vortex system are investigated via a core detection technique. Emphasis is placed on the mutual induction effects between the two main vortices rolling up from the pressure and suction sides of the flap edge. A simple heuristic formula that relates the far-field noise spectrum and the cross-spectrum of the unsteady vortical positions is developed.
\end{abstract}

\section{Introduction}

$\mathrm{T}^{\mathrm{H}}$ HE use of space/time resolved CFD simulations in the prediction of airframe noise sources is becoming more prevalent in the design of low-noise devices for high-lift systems ${ }^{1,2,3}$ and landing-gears. ${ }^{4}$ Very recently, under NASA's Environmentally Responsible Aviation (ERA) project, ${ }^{5,6,7}$ numerical simulations have been used as an initial screening tool to downselect flap and gear noise reduction concepts for further experimental studies and flight tests, ${ }^{8}$ with a positive impact on the development costs of original low-noise concepts and designs. More importantly, the high-fidelity computational simulation for the baseline untreated configuration can be used to gain a deeper understanding of the airframe noise sources and noise generation mechanisms associated with aircraft high-lift devices and landing gear. Such a fundamental insight would ultimately lead to the design and development of more efficient noise reduction technologies.

The noise generated at the side edges (tips) of aircraft flaps, which is a major component of airframe noise during landing, has received considerable attention during the past two decades. ${ }^{9,10,11,12,13,14,15,16}$ Most of these studies involved a generic, straight edge flap tip geometry in a quasi two dimensional wing $/$ high-lift system configuration. As shown in Fig. 1, the emerging picture of the flow field is that of a dual vortex system with the stronger vortex originating on the side wall at the bottom (pressure) edge and the weaker vortex residing near the upper edge on the top (suction) surface of the flap. Khorrami and Singer ${ }^{11}$ have conjectured that the noise generation mechanism(s) can be attributed to side-edge flow characteristics such as large-scale fluctuations being supported by the free-shear layer from the flap bottom edge and a postmerged vortex downstream of the flap mid-chord region, turbulent boundary layer convection over sharp

\footnotetext{
*Technical Director, Aeroacoustics, Aerospace, member AIAA.

$\dagger$ Application Engineer.

$¥$ Technical Director, Aerospace, Senior member AIAA.

$\S$ Aerospace Engineer, Computational AeroSciences Branch, Associate Fellow AIAA.
} 
edges, vortex merging, and vortex breakdown. However, a concrete cause and effect relationship between the far-field noise and any of the conjectured flow features remains elusive. The present study sheds some light on this very challenging problem by trying to extract information on relevant flow features from a pre-existing high-fidelity, fully vetted simulation and establish the underlying connection between side edge vortex dynamics and sound radiation.

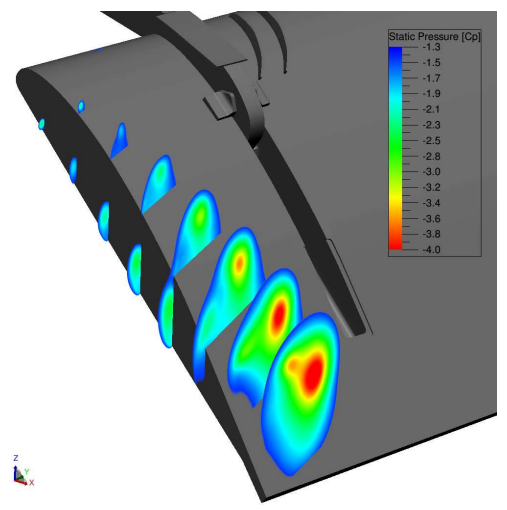

Figure 1. Flap side-edge dual-vortex system.

The paper is organized as follows. The aircraft model and flow conditions are described in Section II. A brief introduction of the employed CFD/CAA approach is provided in Section III. Sections IV and V are devoted to demonstrating the connections between the far-field noise and the side-edge aerodynamic force, and between the side-edge force and the vortex kinematics, respectively. The main findings of the present analyses are summarized in the conclusion section, together with a discussion on further analyses required to answer some of the unresolved issues and remaining questions.

\section{Aircraft Geometry Model and Flow Conditions}

The simulated model geometry corresponds to an 18\%-scale, semi-span, high-fidelity reproduction of a Gulfstream aircraft shown in Fig. 2. A full description of the aircraft model, including the surface distribution of steady pressure ports and unsteady transducers on various components, is provided in Refs. 5 and 6 . The model geometry consists of a fuselage, a wing, a flap, a flow-through nacelle, a pylon, and a main landing gear. The aircraft model landing configuration of $39^{\circ}$ flap deflection with the main gear removed was used for the present flap side edge noise study. ${ }^{7}$ The same configuration was also used in the past to perform an extensive computational investigation focused on mesh resolution and solution convergence. ${ }^{7,8}$
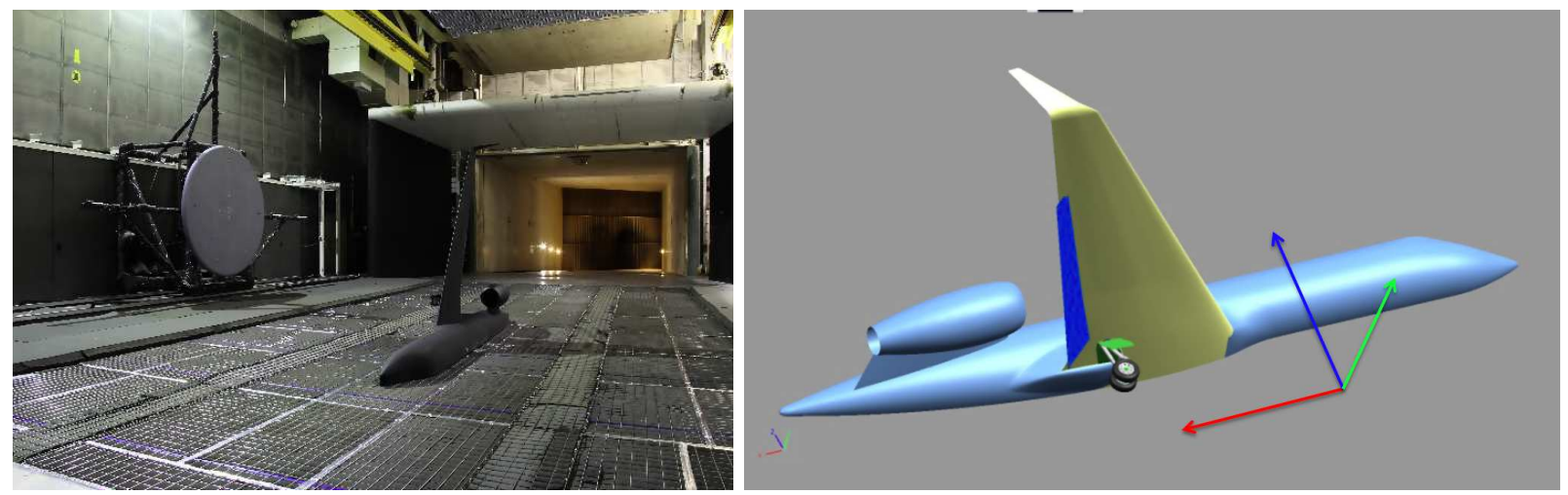

Figure 2. 18\%-scale, semi-span model of a Gulfstream aircraft. NASA LaRC $14 \times 22$ foot wind tunnel on the left, and CAD model on the right.

Simulations were conducted with inlet reference flow variables set to match conditions measured during the wind tunnel tests conducted in the NASA Langley Research Center (LaRC) 14- by 22-Foot Subsonic 
Tunnel. A freestream Mach number of 0.2 with $3^{\circ}$ angle of attack was used, corresponding to a Reynolds number of $3.40 \times 10^{6}$ based on the $18 \%$-scale model Mean Aerodynamic Chord (MAC) of $0.782 \mathrm{~m}$. A complete account of the computational setup and solution approach is provided in Ref. 7 .

As discussed in Ref. 7, the outboard flap side edge has a cavity that nearly spans the entire chord length. In the actual aircraft, this cavity houses a bulb seal that prevents metal to metal contact during flap retraction. On the other hand, the inboard flap tip has a straight edge geometry similar to those generic configurations used in past fundamental studies. Since the focus of the present work is on the aeroacoustic mechanisms taking place in canonical flap side edge flows, only noise from the inboard side edge is targeted. For swept and tapered flaps such as the one considered here, the larger inboard side edge generates lower frequency noise relative to the smaller outboard side edge, as illustrated by the two beamforming maps presented in Fig. 3. These maps were constructed using numerical noise signals extended to the far-field from near-field surface data by using a Ffowcs Williams-Hawkings (FW-H) solver.

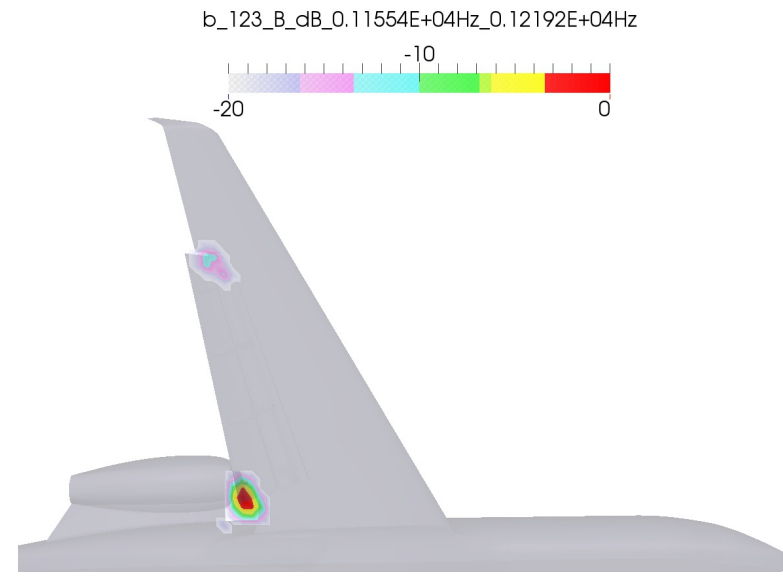

(a) $1.25 \mathrm{kHz}$

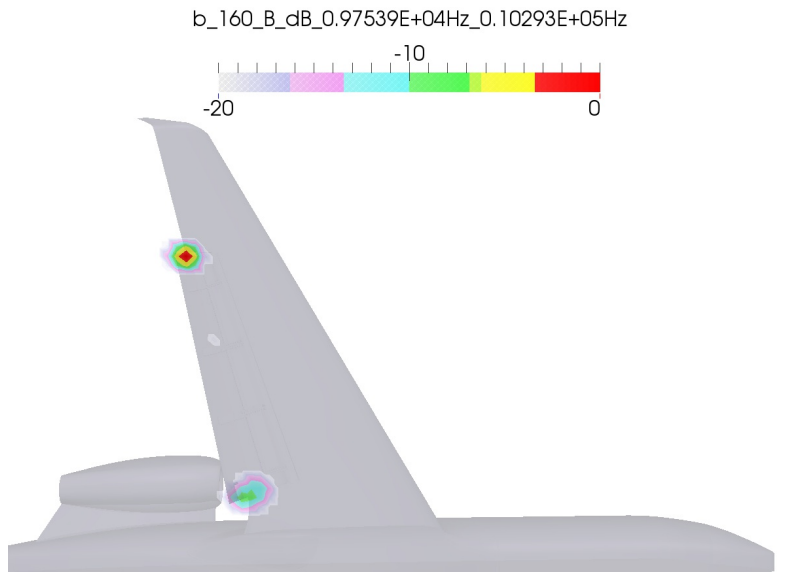

(b) $10 \mathrm{kHz}$

Figure 3. Beamforming maps in $1 / 12$ th octave bands centered at $1.18 \mathrm{kHz}$ and $10 \mathrm{kHz}$ (levels normalized by the maximum value for each band).

The far-field noise analysis performed in the present study is based on the noise signal computed at one single microphone with coordinates $(-0.857,1.706,-6.468)$ normalized by the MAC in a reference system with origin in the mid-chord point of the inboard flap side edge, and aligned with the aircraft reference system sketched in Fig. 2.

For a more targeted analysis of the vortex dynamics and associated noise, the flap surface in the vicinity of the side edge is segmented into 10 spanwise strips along the chord, with each segment comprised of three surfaces, one on the pressure side, one on the suction side, and one on the side wall. The individual segments are highlighted with different colors in Fig. 4 and enumerated from 1 (leading-edge) to 10 (trailing-edge).

\section{CFD/CAA Numerical Model}

The flow solver PowerFLOW ${ }^{\circledR} 5.0$ was used to generate the computational data set for the present study. The physical core of the software solves the Boltzmann equation for the distribution function $f(\mathbf{x}, t, \mathbf{v})$ on a hexahedral mesh automatically generated around bodies consisting of one or more solid parts. The Boltzmann equation is solved by discretizing the particle velocity space into a prescribed number of values. Once the distribution function is computed, the macroscopic flow quantities, density and linear momentum, are determined through discrete integration. All the other quantities are determined through thermodynamic relationships for an ideal gas.

Solving the lattice Boltzmann equation is equivalent to performing a Direct Numerical Simulation (DNS) of the Navier-Stokes equations in the limits of the dynamic range (Mach number) that can be accurately covered by the number of discrete particle velocity vectors, and in the limits of the lattice resolution required to capture the smallest scales of turbulence. For high Reynolds number flows, turbulence modeling can be incorporated into the lattice Boltzmann method (LBM) scheme by solving a variant of the renormalization group (RNG) $k-\epsilon$ model for the unresolved scales. ${ }^{17}$ This approach is referred to as LBM Very Large Eddy 


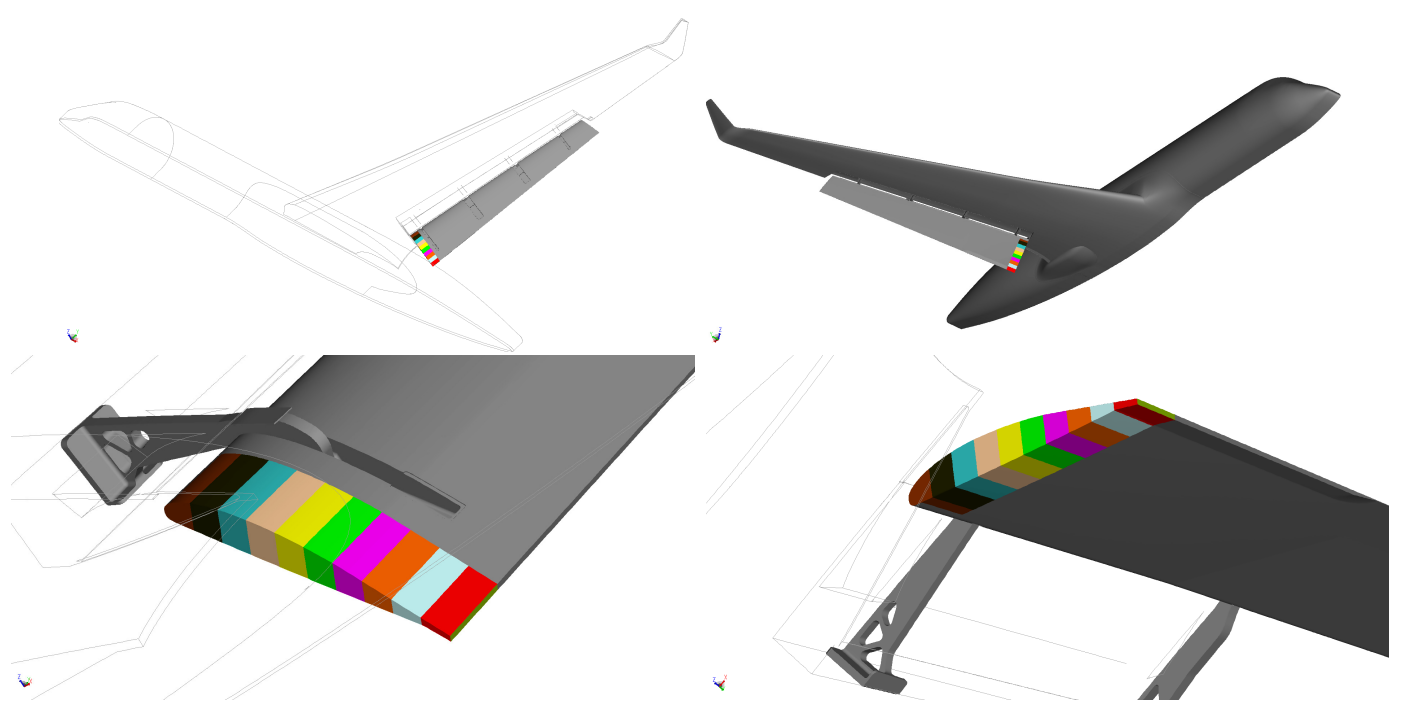

Figure 4. Segmentation of the inboard flap side edge.

Simulation (LBM-VLES).

Because resolving the wall boundary layer by using a Cartesian mesh approach down to the viscous sub-layer in high Reynolds number applications is prohibitively expensive, a wall function approach is used in PowerFLOW to model boundary layers on solid surfaces. The wall function model is an extension of the standard formulation, ${ }^{18}$ but it includes the effects of favorable and adverse pressure gradients and accounts for surface roughness through a length parameter. ${ }^{19}$

The LBM scheme is solved on a grid composed of cubic volumetric elements (voxels). Variable resolution by a factor of two is allowed between adjacent regions. Correspondingly, the time step is varied by a factor of two between two adjacent resolution regions. Solid surfaces are automatically facetized within each voxel intersecting the wall geometry by using planar surface elements (surfels). For the no-slip and slip wall boundary conditions at each of these elements, a boundary scheme ${ }^{20}$ is implemented, based on a particle bounce-back process and a specular reflection process, respectively. Therefore, very complex arbitrary geometries can be treated automatically.

The local character of the LBM scheme allows an efficient parallelization of the solver. Moreover, given the intrinsic low dissipative properties of the scheme, ${ }^{21}$ LBM is well suited for aeroacoustic simulations and, in particular, for airframe noise applications. ${ }^{22,23,7,24}$

The far-field noise is computed through an integral extrapolation based on a solid FW-H acoustic analogy formulation. A forward-time solution ${ }^{25}$ of the FW-H equation based on Farassat's formulation 1A, ${ }^{26}$ generalized to a permeable integration surface, ${ }^{27}$ is used. The freestream convective effects are taken into account directly in the integral formulation, as described in Refs. 28 and 29. In the present study, non-linear volume sources are neglected and integrations are carried out on the solid surface of the aircraft model. This approach is usually preferred for airframe noise computations for two reasons: the space-time resolution on the model surface is higher than those in the field, thus allowing higher frequency noise predictions; and no spurious effects are introduced due to strong coherent turbulent fluctuations convected through a permeable integration surface.

\section{Connection between Side-Edge Noise and Aerodynamic Forces}

This section is focused on the relationship between the far-field noise computed at the reference microphone and the unsteady aerodynamic forces induced by the tip vortex dynamics.

As a first step, we compute the noise contribution of each tip segment separately using the FW-H analogy and compare it to the overall side-edge noise. Figure 5 shows third-octave band noise spectra for the 10 segments at the inboard tip of the flap. On the left image, noise levels have been normalized by the overall sound pressure level of the entire flap tip, whereas on the right image, levels of each band have been 
normalized by the corresponding band level of the noise generated by the entire flap tip. The figure on the left clearly shows that the relative contribution to the overall noise increases along the chord; the increment in noise level is accompanied by a reduction of the frequency at which the maximum noise contribution takes place. The chordwise increment in noise level can be explained by an increase in the vortex strength due to the continuous roll-up of vorticity sheets associated with the shear layers originating from the lower and upper edges. The decrease of the dominant noise frequency can be explained by the increase in both vortex size and vortex distance from the flap top surface, which results in a reduction of the pulsation frequency around the equilibrium location. ${ }^{14}$ If this is the case, the tip vortex system is not moving rigidly around its equilibrium position, but each vortex section is characterized by a dominant frequency, thus resulting in a deformation of the core line while preserving the vortex coherent structure. The figure on the right confirms that the relative contribution to the overall noise increases from the leading to the trailing edge of the flap. At high frequencies, the relative contribution from each segment tends to become less sensitive to frequency variations. This may be explained by the fact that higher frequency sources are most likely associated with small-scale flow structures that tend to have a more universal character, e.g., wall-bounded turbulence, or small-scale vortex filaments with similar structure along the chord. Notice that the relative contributions of segments 8 and 9 exhibit an opposite frequency trend, maybe due to the different source mechanisms taking place after merging of the two vortices.
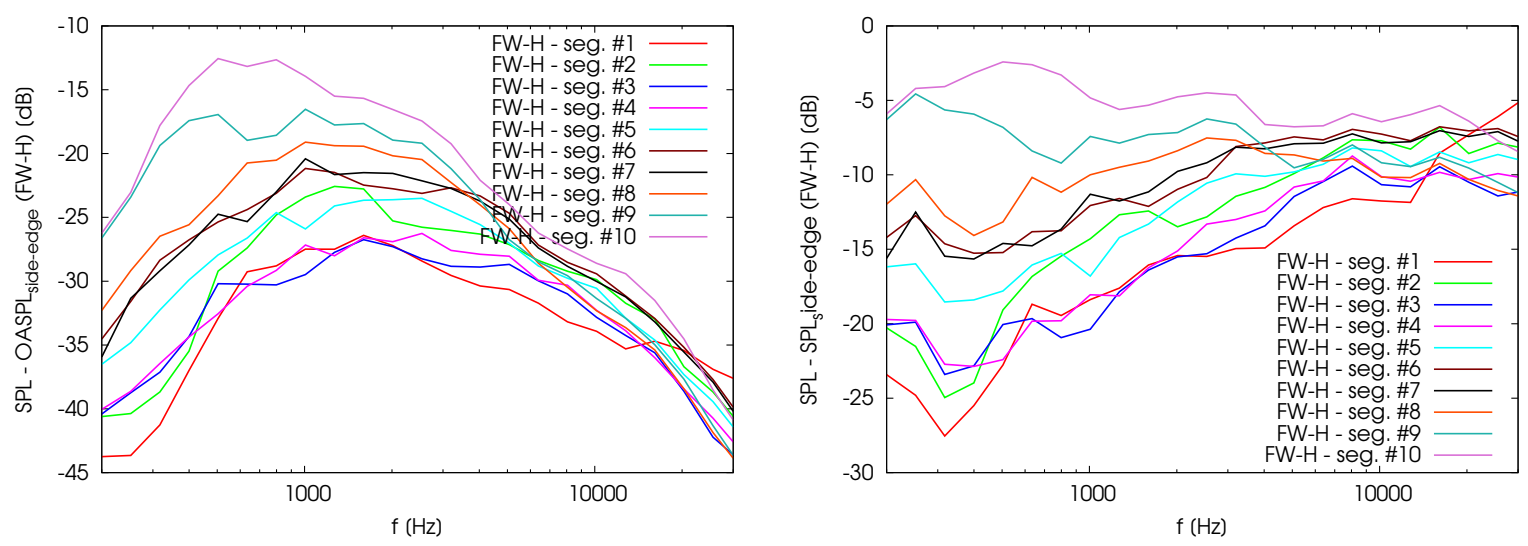

Figure 5. Relative noise contribution of the different side-edge segment to the overall flap side-edge noise.

As a second step, we seek to understand how well the flap side-edge noise source can be modelled as a compact dipole. Based on the inboard tip dimensions, it is certainly geometrically compact with respect to the microphone distance. Therefore, the main question would be for what frequency range the side-edge, or its segments, are acoustically compact. A compact dipole noise formula can be derived directly from the dipole term of the FW-H analogy (see for instance Ref. 30) in the geometrical and acoustic far-field limit. The acoustic pressure $p^{\prime}$ at the point $\mathbf{y}$ and time $\tau$ is given by:

$$
p^{\prime}(\mathbf{y}, \tau)=\frac{\dot{\mathbf{F}}(\mathbf{x}, t) \cdot \hat{\mathbf{r}}}{4 \pi c_{\infty} r\left(1+M_{r}\right)^{2}},
$$

where $\dot{\mathbf{F}}$ is the time derivative of the aerodynamic force on the body, $\mathbf{x}$ is the dipole source location, $\mathbf{r}=\mathbf{y}-\mathbf{x}$ is the radial vector, $r=|\mathbf{r}|$ is the source to observer distance, $\hat{\mathbf{r}}=\mathbf{r} / r$ is the unit radial vector, $M_{r}=M_{\infty} r_{x}$ is the projection of the freestream Mach number in the radiation direction, $c_{\infty}$ is the speed of sound and $t=\tau-r / c_{\infty}$ is the emission time.

The components of the aerodynamic force are extracted for each segment of the tip and used to compute the corresponding compact dipole noise. Each dipole is assumed to be located at the midpoint of the lateral face of the segment. Then, the overall aerodynamic force acting on the entire tip is used to compute the noise generated by the overall flap side-edge by assuming that the dipole is located at the midpoint of the lateral face. Spectra and overall sound level of the compact dipole noise signals are compared to the reference FW-H in Fig. 6. As can be seen, the compact dipole formula matches the FW-H overall sound levels to within $0.2 \mathrm{~dB}$. For all segments the agreement is almost uniform over the entire frequency range of interest, say, up to $30 \mathrm{kHz}$, with only a slight worsening taking place at the highest frequencies. This is quite surprising 
because the chordwise extension of each segment is about $25 \mathrm{~mm}$, whereas the acoustic wavelength at $30 \mathrm{kHz}$ is about $11 \mathrm{~mm}$. The agreement noted here is not proof of source compactness, as it is associated with only one microphone location and does not take into account diffraction effects. Further study is necesssary to ascertain the causes of this behavior.
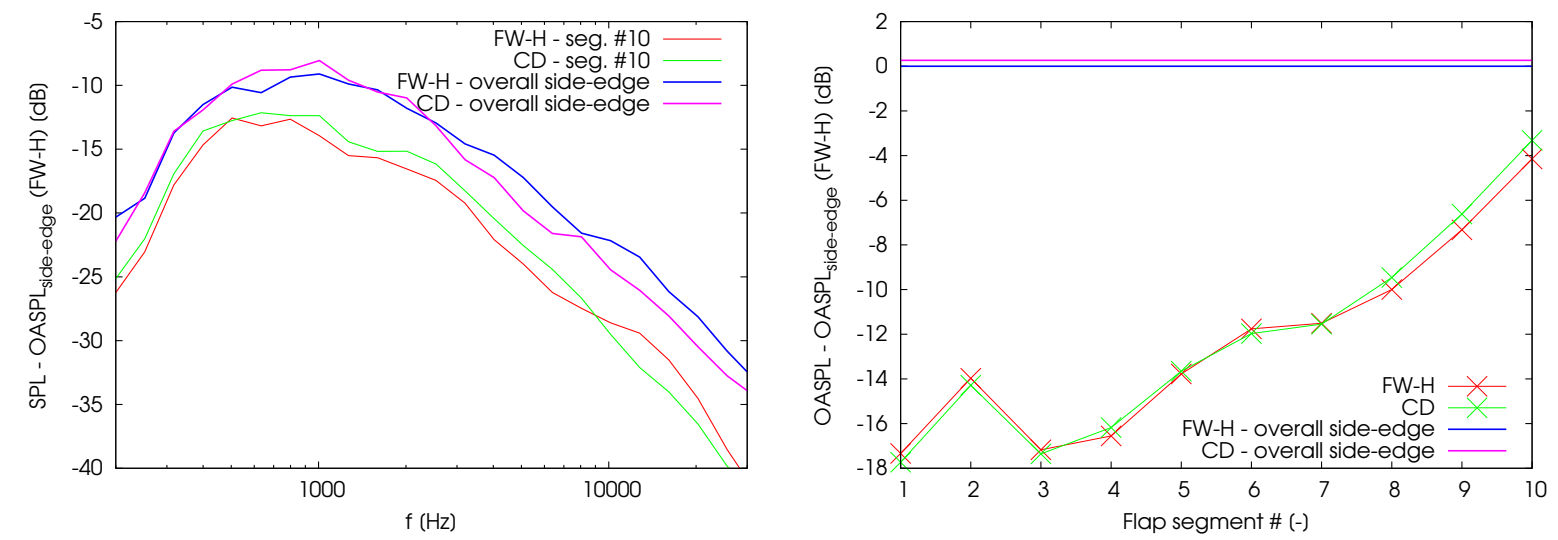

Figure 6. Comparison between FW-H and Compact Dipole (CD) noise results. On the left, third-octave band sound pressure levels, on the right overall sound pressure levels. All levels normalized by the overall side-edge sound pressure level computed using the FW-H formulation.

Finally, integrated noise levels generated by the pressure, lateral and suction sides of the flap side-edge are reported in Table 1. The overall side-edge noise levels computed using the FW-H analogy and the compact dipole formulation are practically the same.

\begin{tabular}{l|ll} 
Face & FW-H & Compact dipole \\
\hline Entire side-edge & 79.797 & 80.063 \\
Pressure side & 71.438 & - \\
Lateral face & 57.058 & - \\
Suction side & 77.089 & -
\end{tabular}

Table 1. Overall sound pressure level for different faces of the side-edge.

The good agreement between FW-H and compact dipole noise results allows us to investigate the noise generation mechanisms taking place in the flap side-edge by focusing on the aerodynamic forces and thus on the vortex dynamics about the side-edge. This is one of the important outcomes of the present study.

\section{Connection between Aerodynamic Forces and Tip-Vortex Kinematics}

In this section we target the relationship between the unsteady aerodynamic forces induced by the tip vortex dynamics and the oscillation of the vortex core lines around their equilibrium position.

Figure 7 shows instantaneous and time-averaged total pressure contours in two spanwise planes cutting through the flap side edge, one located at the mid-chord, the other closer to the trailing edge. The typical pattern of two vortices rolling up from the pressure and suction sides of the flap can be observed in the mid-chord plane. Downstream, the two vortices merge producing a significantly larger semi coherent vortex on the suction side. As will be shown next, a more detailed analysis revealed that the two vortices are able to preserve a certain coherent and independent structure along the full extension of the flap side-edge.

To investigate the connection between vortex kinematics and aerodynamic force acting on the sideedge, as a first step, the instantaneous position of the two vortices is extracted on planes that are nearly perpendicular to the flap chord line and located at the mid point of the flap segments \#3 to \#10. Figure 8 shows an image of the vortex system extracted from the time-averaged flow solution, together with the planes used to determine the positions of the cores.

A transient flow of $2.04 \times 10^{-1} \mathrm{~s}$ duration sampled at $101.5 \mathrm{kHz}$ was used for vortex core detection purposes. Among the several techniques employed, the approach that provided a better tradeoff between CPU time 

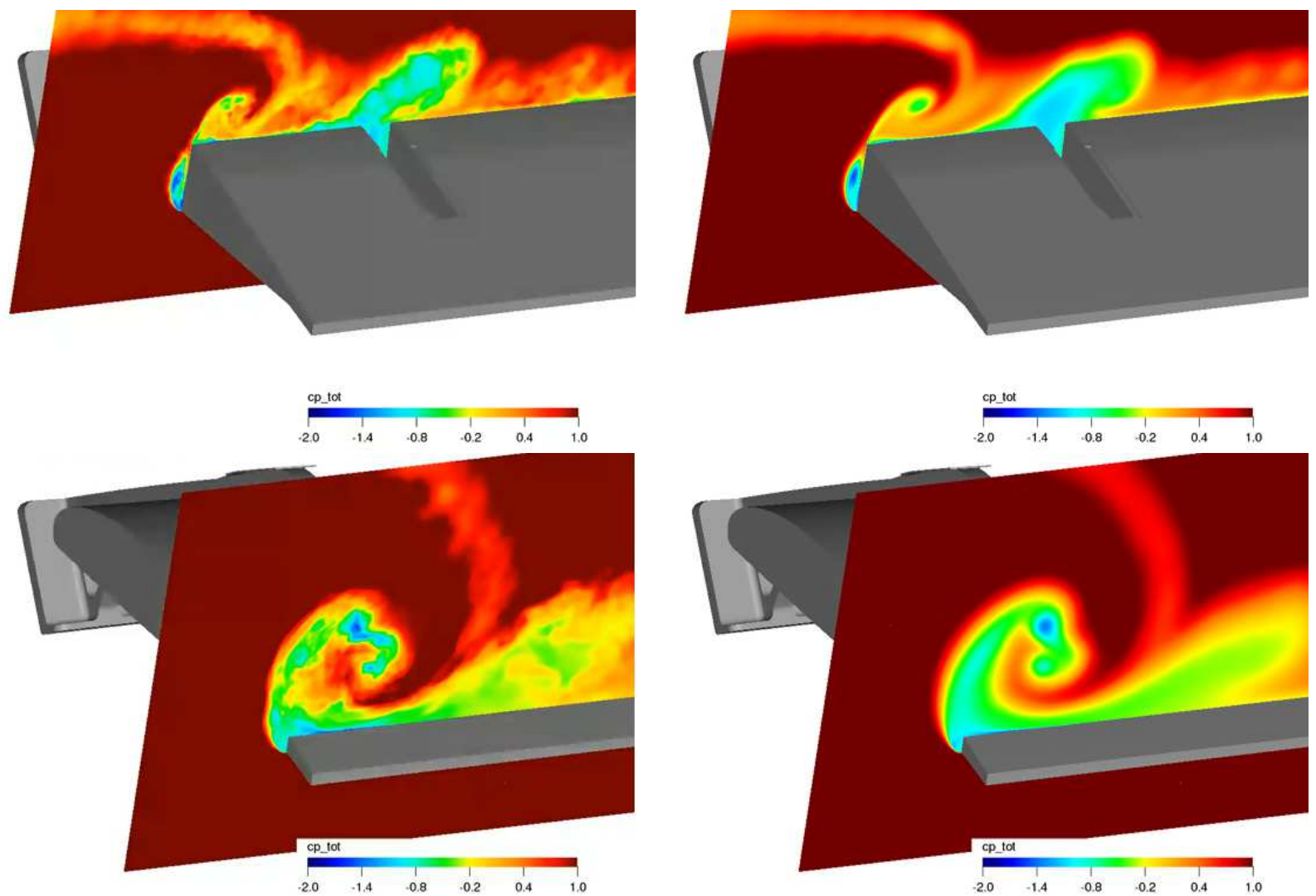

Figure 7. Tip-vortex system in two planes along the chord of the flap. Instantaneous (left) and time-averaged (right) contour levels of the total pressure.

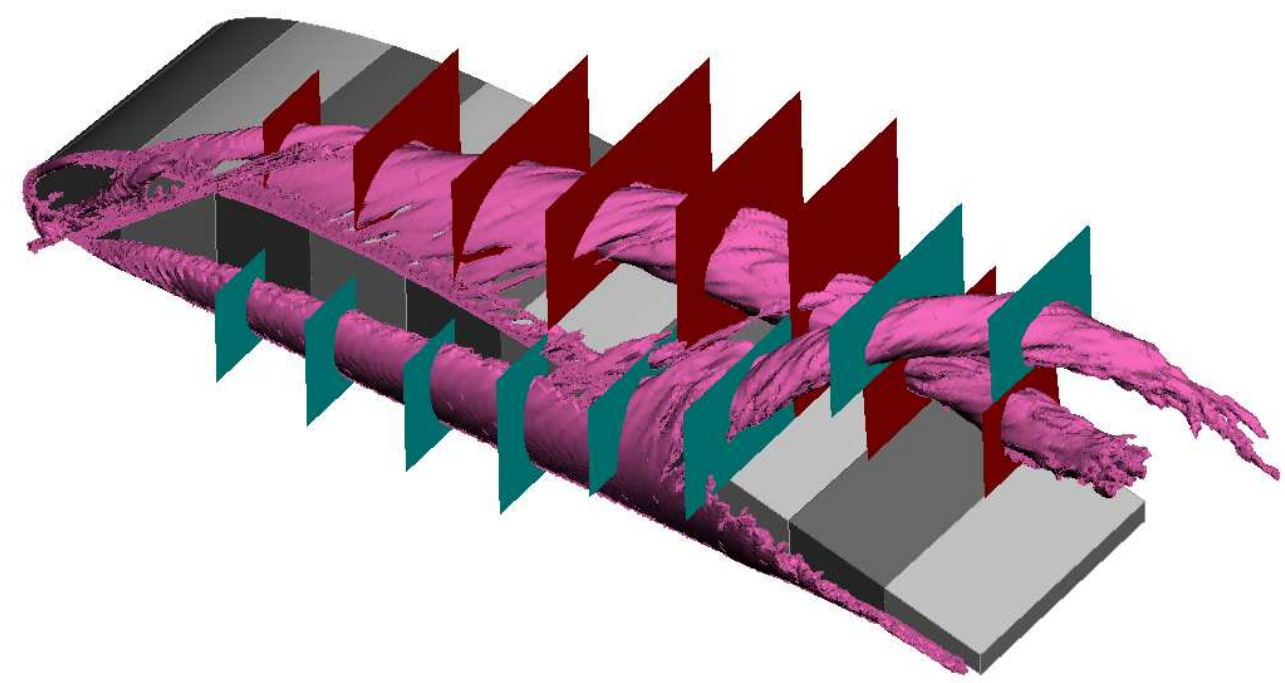

Figure 8. Iso-surface of $\lambda_{2}$-criterion and vortex core extraction planes along the flap chord. 
expenditure and reliability of vortex core detection is based on a kinematical model and consists of determining the instantaneous center of rotation of the velocity field in a search plane with normal $\mathbf{n}$. Denoting as $\mathbf{x}_{i}$ one of the $N$ points of the search mesh, the following quantity is computed:

$$
\Upsilon\left(\mathbf{x}_{i}\right)=\frac{1}{N-1} \sum_{\substack{j=1 \\ j \neq i}}^{N} \frac{\left(\mathbf{x}_{j}-\mathbf{x}_{i}\right) \times\left(\mathbf{v}_{j}-\tilde{\mathbf{v}}\right)}{\left|\mathbf{x}_{j}-\mathbf{x}_{i}\right|\left|\mathbf{v}_{j}-\tilde{\mathbf{v}}\right|} \cdot \mathbf{n}, \quad \text { with } \quad \tilde{\mathbf{v}}=\frac{1}{N} \sum_{j=1}^{N} \mathbf{v}_{j}
$$

where $\mathbf{v}_{j}$ is the flow velocity at the $j$ th point and $\tilde{\mathbf{v}}$ is the space-averaged velocity over the search plane. The vortex core is assumed to be located at the point where the quantity $\Upsilon$ is maximum.

As a next step, an analytical model based on conformal mapping applied to each extraction plane separately is employed to derive the aerodynamic force generated by the vortex core movement in each plane. This approach is aimed at defining the vortex trajectory in a functional form that can be better correlated to the computed aerodynamic force and noise instead of a merely geometrical functional, based, for instance, on the distance between the vortices and a specific point on the side-edge profile. The intersection between each flap segment and the vortex core extraction plane is approximated by a semi-infinite rectangular edge. Subsequently, a conformal mapping of the Schwarz-Christoffel family is used to map the semi-space $\zeta$ above an infinite plane into the physical space $z$ around the blunt edge. For such a simple geometry, the transformation can be determined in closed form. Denoting as $A$ and $B$ the lower and upper sharp edges of the side-edge profile, which are mapped into the singular points $(-1,0)$ and $(1,0)$ in the semi-infinite plane, respectively, the mapping $z=z(\zeta)$ is defined as:

$$
z=z_{0}+\left\{\frac{-2}{\pi}\left[\zeta \sqrt{1-\zeta^{2}}+\mathrm{i} \ln \left(\zeta+\mathrm{i} \sqrt{1-\zeta^{2}}\right)\right]-1\right\} t \mathrm{e}^{\mathrm{i}\left(\pi / 2+\theta_{0}\right)}
$$

where $z_{0}=x_{0}+\mathrm{i} y_{0}$ is the mid point of the side segment $\overline{A B}, t$ is the thickness of the side segment, and $\theta_{0}=-\arcsin \left[\left(x_{B}-x_{A}\right) /(2 t)\right]$ denotes the orientation of the side-edge profile. The inverse mapping is not defined in closed form, and a procedure based on a simplex algorithm is used to evaluate the relationship $\zeta=\zeta(z)$. This is used to determine the instantaneous position of the two vortices in the mapped plane, say $\zeta_{v}$. Since the conformal mapping is singular at the two poles $\zeta= \pm 1$, resulting in infinite velocities at the corners induced by the two vortices and their images, a Kutta-like condition can be enforced in the system by introducing two vortices at the locations $( \pm 1+i \varepsilon)$, where $\varepsilon=0.01$. The instantaneous circulation of these two vortices is set in such a manner that combined they cancel the velocity induced by the physical vortices at the poles in the $\zeta$ plane. This procedure acts as a regularization of the solution by smoothing the velocity past the corners in the physical domain; numerical tests revealed that the parameter $\varepsilon$ has a weak effect on the vortex-induced force. The potential field induced by the system of two physical vortices $v$, two corner vortices $w$, and their respective images can be represented by:

$$
W(\zeta, t)=-\sum_{j=1}^{2} \frac{\mathrm{i} \Gamma_{v j}}{2 \pi} \ln \left(\frac{\zeta-\zeta_{v j}(t)}{\zeta-\zeta_{v j}^{*}(t)}\right)-\sum_{k=1}^{2} \frac{\mathrm{i} \Gamma_{w k}}{2 \pi} \ln \left(\frac{\zeta-\zeta_{w k}(t)}{\zeta-\zeta_{w k}^{*}(t)}\right)
$$

where the vortex circulations $\Gamma_{v}$ are evaluated by computing the flux of vorticity in the direction normal to the extraction plane over a circular area around the vortex, whereas, as already stated, the vortex circulations $\Gamma_{w}$ are computed by cancelling at every time step the velocity $V(\zeta)$ at the poles. Throughout, the superscript * denotes complex conjugate. The velocity in the physical domain can be determined in a straight forward manner by exact analytical differentiation (not shown) using the following relationship:

$$
V(z, t)=\frac{\partial W(\zeta, t)}{\partial \zeta} / \frac{\partial z}{\partial \zeta}
$$

Finally, the gauge pressure distribution on the side-edge profile can be computed through the generalized Bernoulli formula:

$$
\frac{p^{\prime}(z, t)}{\rho_{\infty}}=-\frac{V V^{*}}{2}-\operatorname{Re}\left(\frac{\partial W}{\partial t}\right)
$$

which can be integrated over the physical spanwise extension $s$ of the flap segment to determine an aerodynamic force comparable to the force computed from the CFD solution. 
Figure 9 shows, for each flap segment, the cloud of vortex positions on the left; and the corresponding spectrum of the force magnitude on the right, normalized by $F_{\text {ref }}=0.5 \rho_{\infty} V_{\infty}^{2} c s$. The vertical black line in the images showing the two vortices and the side-edge profile denotes the spanwise extension $s$ of each flap segment used for the analytical force computation based on Eq. 6. For the remainder of this paper, vortex \#1 is the vortex shed and fed from the pressure (lower) side of the flap. Observed that, although there are noticeable differences between the analytical and the CFD force spectra, the frequency trend is correctly captured by the analytical force, in particular for those flap segments where the two vortices are not too far from the edge (solid surface). The differences can be partially explained by considering that the analytical model is based on a two-dimensional vortex induction formulation, and that three-dimensional induction effects due to the vortex filament cannot be neglected when the vortex distance from the side-edge is not smaller than the length of the vortex filament. Hence, it has been shown here that analytical models of flap side-edge noise based on conformal mapping can be used to explain some of the trends, ${ }^{14}$ but can also lead to quantitatively wrong results.
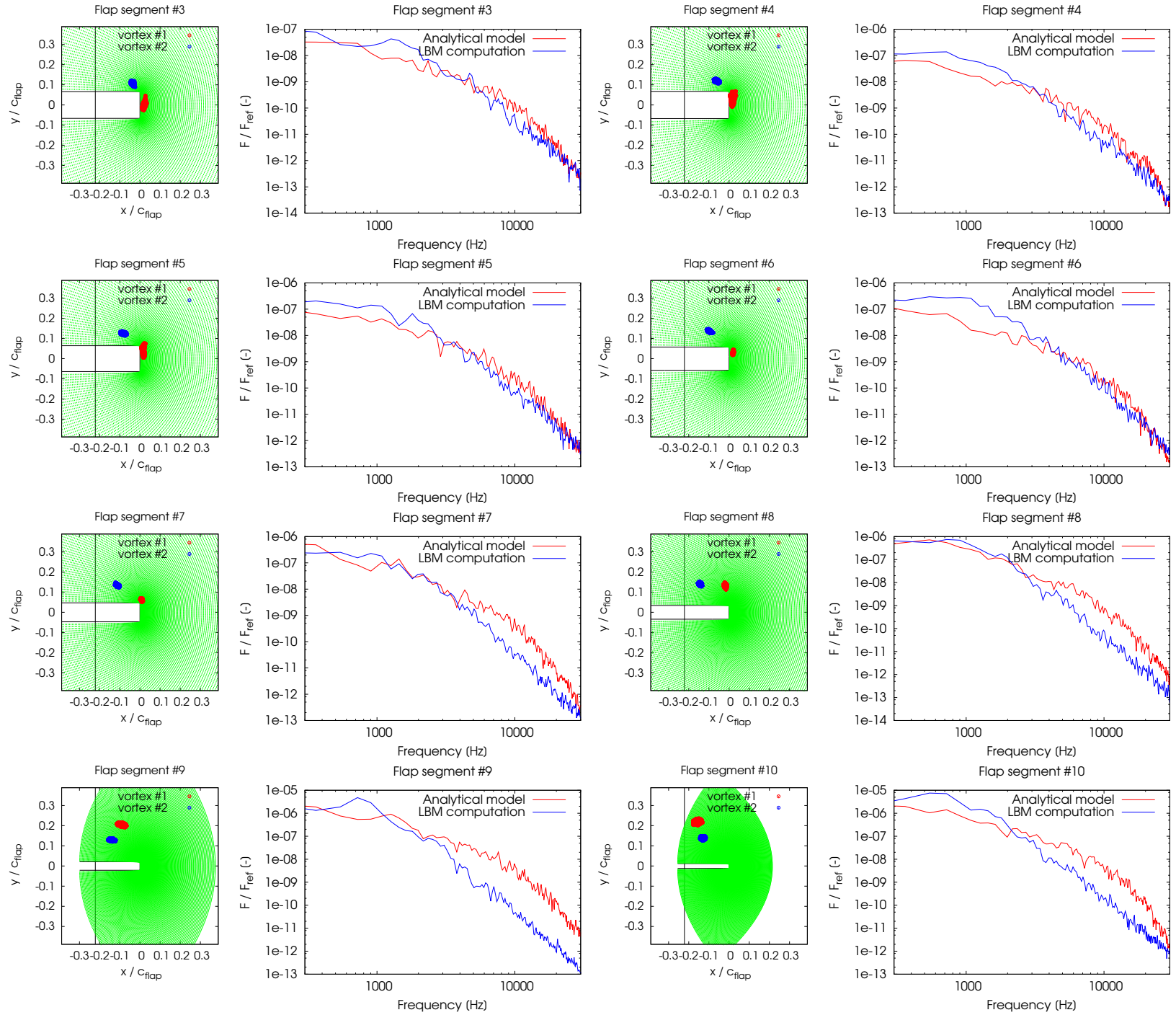

Figure 9. Cloud of vortex locations and spectrum of the aerodynamic force magnitude, for flap segments from $\# 3$ to $\# 10$.

The force spectra plotted in Fig. 9 seem to support our hypothesis that the aerodynamic force acting on the side-edge is almost entirely due to the movement of the two vortices. Further confirmation of this hypothesis can be obtained from a direct comparison between the farfield noise spectrum $S_{p p}$ and a statistical parameter related to vortex location. Among several quantities extracted from the vortex kinematics, the best correlation with the farfield noise was found by using the cross-spectrum between the time-varying 
positions of the two vortices in the direction normal to the edge (denoted as $y$ in Fig. 9), identified as $S_{12} y y$. Figure 10 shows how a simple scaling law $S_{p p}=K+8 \log \left(S_{12 y y}\right)$ is almost homogeneously valid along the flap chord. The constant of proportionality $K$ increases along the chord, most likely caused by the chordwise increase in vortex strength that results from entrainment of vorticity created by shear layer separation at the two sharp edges. This conjecture is confirmed by Fig. 11, where the circulation computed from a timeaveraged flow solution at the vortex core extraction planes and normalized by $V_{\infty} c$ is plotted along the flap chord. Notice that the strength of both vortices undergoes an abrupt increase staring at segment \#7, the segment in which vortex $\# 1$ moved from its position on the side wall to the upper (suction) side. This increase may be related to the change in slope of the radiated noise levels per-segment plotted in Fig. 6.
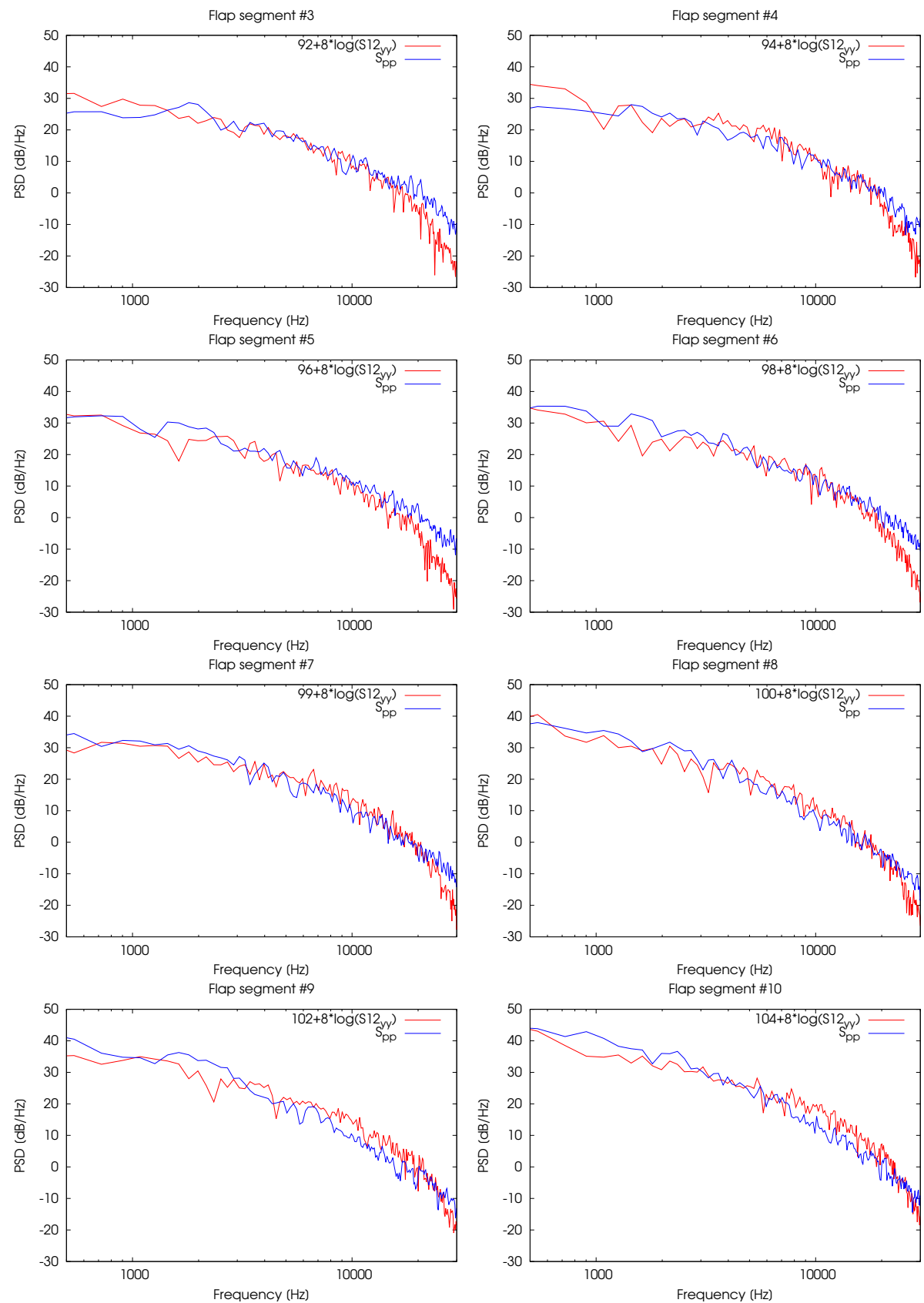

Figure 10. Comparison between far-field noise spectrum and the adhoc scaled cross-spectrum between the two vortices $y$ position, for flap segments from \#3 to \#10.

The chordwise increase in vortex system circulation is certainly related to the chordwise increase in noise generation. As already pointed out in Section IV, the chordwise noise increase is accompanied by a 


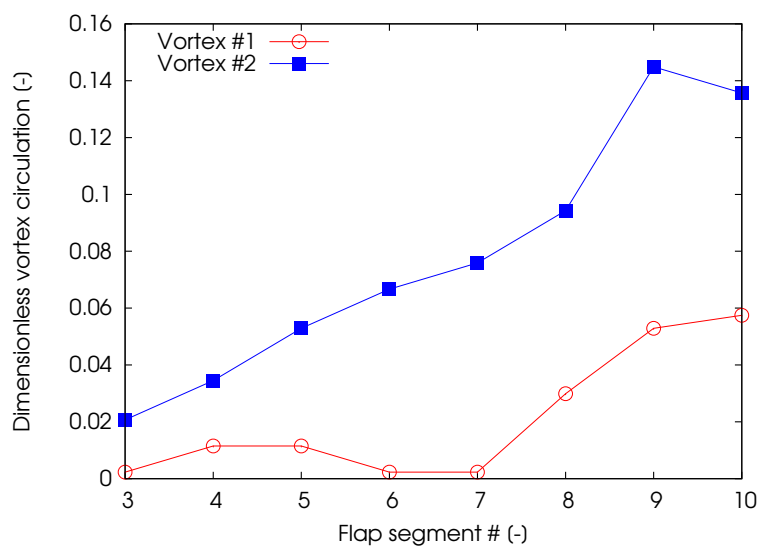

Figure 11. Chordwise vortex circulation.

reduction in the dominant noise frequency. Therefore, according to our kinematical model, the position of the vortex centerline cannot be rigid and should be characterized by significant spatial distortion. Instantaneous snapshots of the reconstructed vortex line (not shown) confirm this hypothesis.

Probability Density Functions (PDF) of the vortex positions along $x$ and $y$ are plotted in Fig. 12. Despite a lack of full convergence due to the small number of samples used, observe that the spanwise fluctuation $(x)$ of vortex \#1 at flap segments \#9 and \#10 displays a significantly flatter distribution, which is associated with a larger lateral (spanwise) movement in vortex position. The unsteady force induced by the fluctuations in the spanwise position of the vortex shed from the pressure side of the flap is therefore likely to be the main mechanism of noise generation once said vortex displaces to the suction side of the flap. Reducing the magnitude of this spanwise movement, by using for instance a tip fence on the suction side, or even modifying the side-edge geometry to fix the vortex position on the pressure side, are possible noise reduction strategies. Another interesting observation is that vortex \#1 undergoes a significant vertical movement at flap segments \#3, \#4 and \#5. Finally, vortex \#2 exhibits more uniform probability distributions along the chord.

An alternate way to describe the statistics of the vortex motion is to compute, for each flap segment, the spectrum of the instantaneous minimum distance from the flap surface. The results are plotted in Fig. 13. For vortex \#1, two frequency ranges of higher fluctuation are evident: the first one from $\sim 300 \mathrm{~Hz}$ to $\sim 800 \mathrm{~Hz}$ on flap segments \#3, \#4 and \#5; the second one from the lowest frequency up to $\sim 1 \mathrm{kHz}$ on flap segments \#8, \#9 and \#10. Note that segment \#8 experiences the most energetic vortex oscillations, possibly caused by incipient vortex merging. These two distinct frequency/location ranges can be associated with the vertical and spanwise movement of vortex \#1 observed in Fig. 12. Finally, similarly to the PDF plots of Fig. 12, vortex \#2 exhibits more uniform spectra along the chord.

\section{Conclusions and Outlook}

The unsteady flow past the flap side edge of a business jet aircraft in landing configuration and the associated noise were investigated through fundamental arguments based on the relationship between aerodynamic noise and forces, and between forces and vortex kinematics. The analysis revealed a clear connection between the far-field noise and the kinematics of two vortices generated from the roll up of the free shear layers originating at the lower and upper edges of the tip. Such a dependency was expected for low frequency noise, dominated by the coherent vortex movement induced by (or related with) macroscopic fluctuations of the flap circulation, but not expected for noise in the high frequency range, which is typically attributed to small-scale turbulent flow motion. The statistical similarity found between noise and vortex movement does not prove definitely that the cause of high-frequency noise is vortex motion, but only that the two mechanisms are related. Further analysis addressing the causality issue is therefore necessary to understand whether the high-frequency oscillations of the vortices around their averaged position is the cause of the force fluctuations, and thus noise, or both are effects of a different source of unsteadiness. Deeper analyses will be performed in the future to shed more light on this mechanism. More precisely, the role of turbulent 

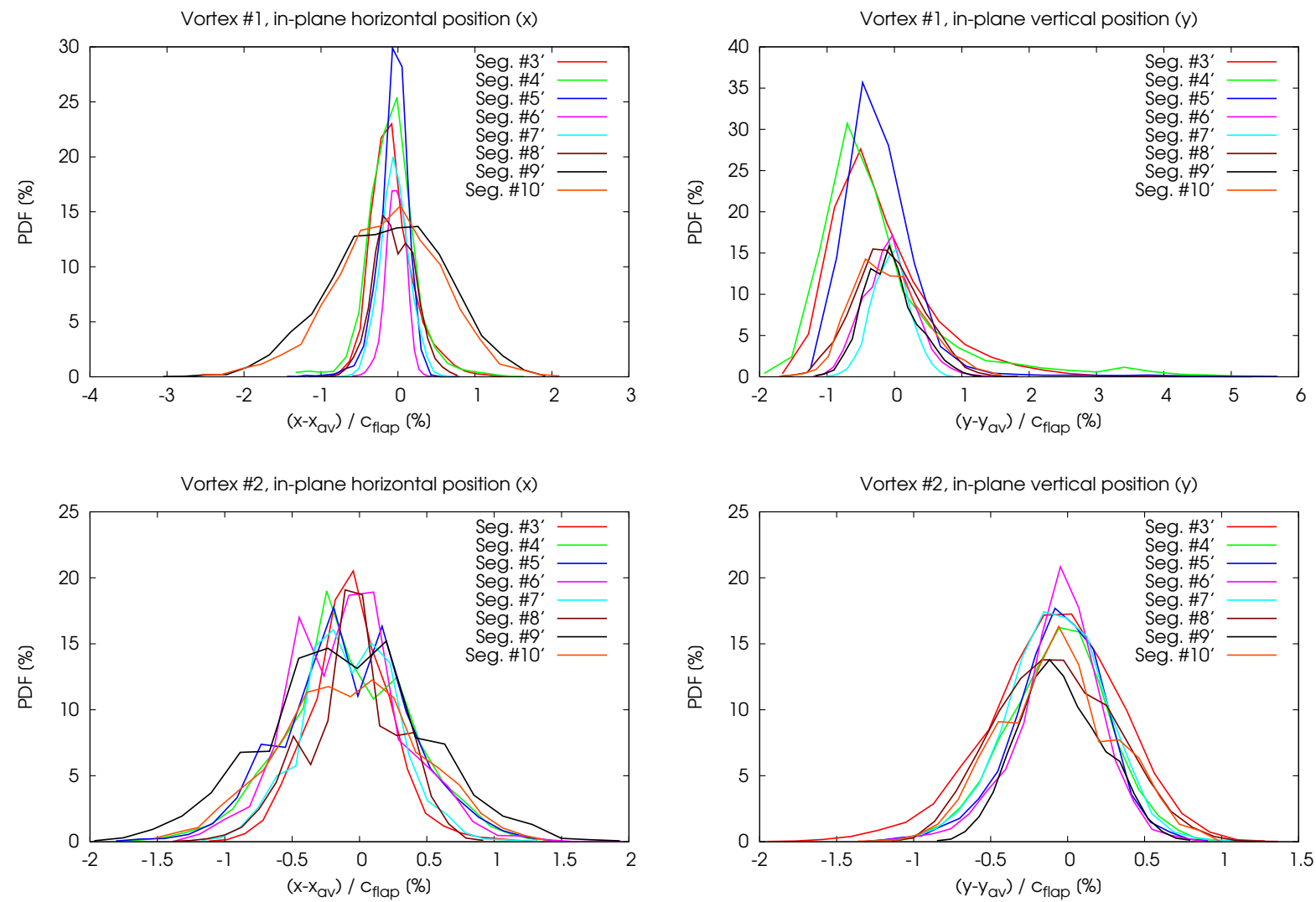

Figure 12. PDF of the positions along the span direction $(x)$ and the chord-normal direction $(y)$ for the two vortices.
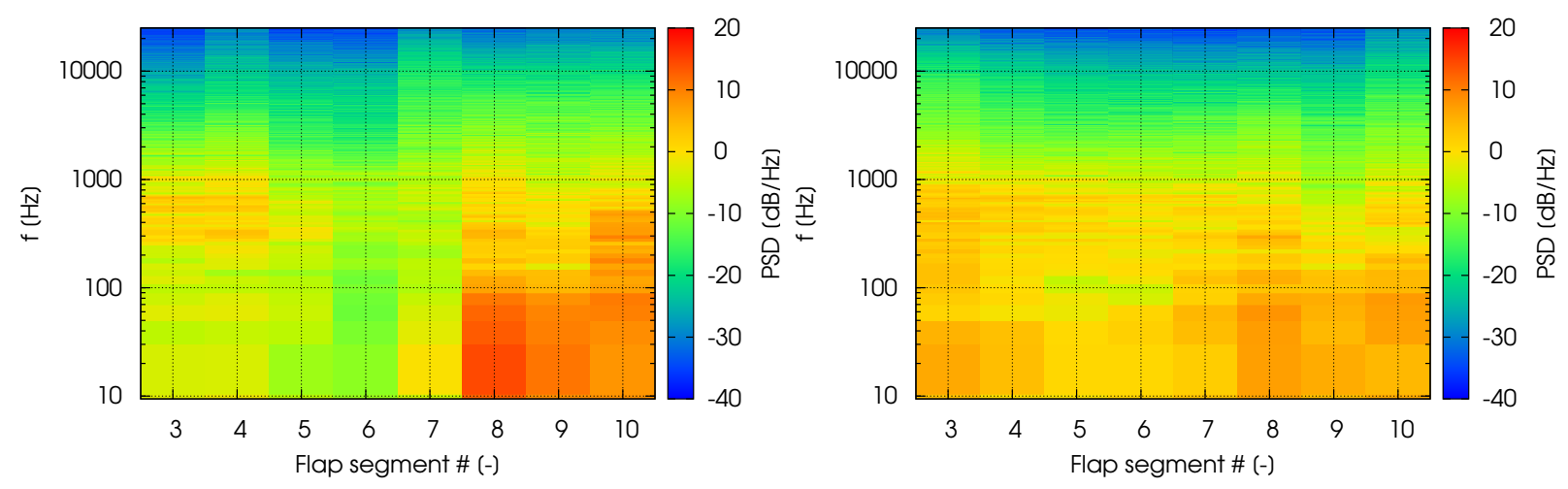

Figure 13. PSD of the vortex distance from side-edge surface along the chord; vortex \#1 (left), vortex \#2 (right). 
fluctuations convected towards the tip along the pressure and suction sides and their ingestion by, and roll up into, the tip vortices needs to be investigated in more detail.

\section{Acknowledgments}

This work was supported by the Environmentally Responsible Aviation (ERA) project under the Integrated System Research Program (ISRP) of NASA.

\section{References}

${ }^{1}$ Choudhari, M. and Khorrami, M. R., "Computational Study of Porous Treatment for an Altered Flap Side-Edge Flowfield," AIA A Paper 2003-3113, 2003.

${ }^{2} \mathrm{Ma}$, Z. and Zhang, X., "Numerical Investigation of Broadband Slat Noise Attenuation with Acoustic Liner Treatment," AIAA Journal, Vol. 47, No. 12, 2009, pp. 2812-2820.

${ }^{3}$ Murayama, M., Yokokawa, Y., Imamura, T., Yamamoto, K., Ura, H., and Hirai, T., "Numerical Investigation on Change of Airframe Noise by Flap Side-edge Shape," AIAA Paper 2013-2067, May 2013.

${ }^{4}$ Rougier, T., "Use of CFD-CAA to Assess Landing Gear Design Changes," Greener Aviation Conference, Clean Sky Breakthroughs and Worldwide Status 2014, Brussels, Belgium, 2014.

${ }^{5}$ Khorrami, M. R., Hannon, J. A., Neuhart, D. H., Markowski, G. A., and de Ven, T. V., "Aeroacoustic Studies of a High-Fidelity Aircraft Model: Part 1-Steady Aerodynamic Measurements," AIAA Paper 2012-2233, 2012.

${ }^{6}$ Khorrami, M. R. and Neuhart, D. H., "Aeroacoustic Studies of a High-Fidelity Aircraft Model: Part 2-Unsteady Surface Pressures," AIAA Paper 2012-2234, 2012.

${ }^{7}$ Khorrami, M. R., Fares, E., and Casalino, D., "Towards Full Aircraft Airframe Noise Prediction: Lattice Boltzmann Simulations," AIAA Paper 2014-2481, June 2014.

${ }^{8}$ Fares, E., Casalino, D., and Khorrami, M., "Computations of Aircraft Airframe Noise reduction Concepts using a Lattice Boltzmann Approach," AIAA Aviation 2015 Conference in Dallas, TX (submitted for publication), 2015.

${ }^{9}$ Hardin, J. C., "Noise Radiation from the Side Edges of Flaps," AIAA Journal, Vol. 18, No. 5, 1980, pp. $549-552$.

${ }^{10}$ Sen, R., "Vortex-Oscillation Model of Airfoil Side-Edge Noise," AIAA Journal, Vol. 35, No. 3, 1997, pp. $441-449$.

${ }^{11}$ Khorrami, M. R. and Singer, B. A., "Stability Analysis for Noise-Source Modeling of a Part-Span Flap," AIAA Journal, Vol. 37, No. 10, 1999, pp. 1206-1212.

${ }^{12}$ Choudhari, M., Lockard, D. P., Macaraeg, M. G., Singer, B. A., Streett, C. L., Neubert, G. R., Stoker, R. W., Underbrink, J. R., Berkman, M. E., Khorrami, M. R., and Sadowski, S. S., "Aeroacoustic Experiments in the Langley Low-Turbulence Pressure Tunnel," NASA TM-2002-211432, 2002.

${ }^{13}$ Brooks, T. F. and Humphreys, W. M., "Flap-Edge Aeroacoustic Measurements and Predictions," Journal of Sound and Vibration, Vol. 261, 2003, pp. 31-74.

${ }^{14}$ Guo, Y. P., "On Noise Reduction by Flap Side Edge Fences," Journal of Sound and Vibration, Vol. 277, No. 1-2, 2004, pp. 369-390.

${ }^{15}$ Koop, L., Ehrenfried, K., and Dillmann, A., "Reduction of Flap Side-Edge Noise: Passive and Active Flow Control," AIAA Paper 2004-2803, May 2004.

${ }^{16}$ Angland, D., Zhang, X., and Molin, N., "Measurements of Flow Around a Flap Side-Edge with Porous Edge Treatment," AIAA Journal, Vol. 47, No. 7, 2009, pp. 1660-1671.

${ }^{17}$ Yakhot, V. and Orszag, S. A., "Renormalization Group Analysis of Turbulence. I. Basic Theory," Journal of Scientific Computing, Vol. 1, No. 1, 1986, pp. 3-51.

${ }^{18}$ Launder, B. and Spalding, D., "The Numerical Computation of Turbulent Flows," Computer Methods in Applied Mechanics and Engineering, Vol. 3, 1974, pp. 269-269.

${ }^{19}$ Fares, E. and Noelting, S., "Unsteady Flow Simulation of a High-Lift configuration using a Lattice Boltzmann Approach," AIAA Paper 2011-869, Jan 2011.

${ }^{20}$ Chen, H., Teixeira, C., and Molvig, K., "Realization of Fluid Boundary Conditions via Discrete Boltzmann Dynamics," International Journal of Modern Physics C, Vol. 9, No. 8, 1998, pp. 1281-1292.

${ }^{21}$ Brès, G. A., Pérot, F., and Freed, D. M., "Properties of the Lattice-Boltzmann Method for Acoustics," AIAA Paper 2009-3395, May 2009.

${ }^{22}$ Casalino, D., Ribeiro, A. F. P., and Fares, E., "Facing Rim Cavities Fluctuation Modes," Journal of Sound and Vibration, Vol. 333, No. 13, 2014, pp. 2812-2830.

${ }^{23}$ Casalino, D., Ribeiro, A. F. P., Fares, E., and Nölting, S., "Lattice-Boltzmann Aeroacoustic Analysis of the LAGOON Landing Gear Configuration," AIAA Journal, Vol. 52, No. 6, 2014, pp. 1232-1248.

${ }^{24}$ Brès, G. A., Freed, D. M., Wessels, M., Noelting, S., and Pérot, F., "Flow and Noise Predictions for the Tandem Cylinder Aeroacoustic Benchmark," Physics of Fluids, Vol. 24, No. 3, March 2012, pp. 036101.

${ }^{25}$ Casalino, D., "An Advanced Time Approach for Acoustic Analogy Predictions," Journal of Sound and Vibration, Vol. 261, No. 4, 2003, pp. 583-612.

${ }^{26}$ Farassat, F. and Succi, G. P., "The Prediction of Helicopter Discrete Frequency Noise," Vertica, Vol. 7, No. 4, 1983, pp. 309-320.

${ }^{27}$ Di Francescantonio, P., "A New Boundary Integral Formulation for the Prediction of Sound Radiation," Journal of Sound and Vibration, Vol. 202, No. 4, 1997, pp. 491-509. 
${ }^{28}$ Brès, G. A., Pérot, F., and Freed, D. M., "A Ffowcs Williams-Hawkings Solver for Lattice-Boltzmann Based Computational Aeroacoustics," AIAA Paper 2010-3711, June 2010.

${ }^{29}$ Najafi-Yazdi, A., Brès, G. A., and Mongeau, L., "An Acoustic Analogy Formulation for Moving Sources in Uniformly Moving Media," Proceeding of The Royal Society of London A, Vol. 467, No. 2125, 2011, pp. 144-165.

${ }^{30}$ Casalino, D. and Jacob, M., "Prediction of Aerodynamic Sounds from Circular Rods via Spanwise Statistical Modeling," Journal of Sound and Vibration, Vol. 262, No. 4, 2003, pp. 815-844. 